\title{
Strank, Karl Josef, Meurers-Balke, Jutta, « ... dass man im Garten alle Kräuter habe ». Obst, Gemüse und Kräuter Karls des Grossen
}

\section{François-Olivier Touati}

\section{OpenEdition}

Édition électronique

URL : http://journals.openedition.org/ifha/1660

DOI : $10.4000 /$ ifha. 1660

ISSN : 2198-8943

Éditeur

IFRA - Institut franco-allemand (sciences historiques et sociales)

\section{Référence électronique}

François-Olivier Touati, « Strank, Karl Josef, Meurers-Balke, Jutta, « ... dass man im Garten alle Kräuter habe ». Obst, Gemüse und Kräuter Karls des Grossen », Revue de I'IFHA [En ligne], Date de recension, mis en ligne le 01 janvier 2008, consulté le 22 septembre 2020. URL : http://journals.openedition.org/ifha/ 1660 ; DOI : https://doi.org/10.4000/ifha.1660

Ce document a été généré automatiquement le 22 septembre 2020.

(CIFHA 


\title{
Strank, Karl Josef, Meurers-Balke, Jutta, "... dass man im Garten alle Kräuter habe ». Obst, Gemüse und Kräuter Karls des Grossen
}

\author{
François-Olivier Touati
}

L'engouement de nos contemporains (continentaux) pour les jardins croiserait-il leur fascination pour un Moyen Âge idéalisé ? Depuis une vingtaine d'années, le fleurissement exponentiel des « reconstitutions » en tout genre, au centre desquelles foisonnent les « carrés » de " simples », est devenu l'incontournable accompagnement paysager des vestiges heureusement préservés, d'abbayes en commanderies, de mottes seigneuriales en vieux bourgs, et même de vénérables ponts. La vision de deux brins de persil et de trois laitues réjouit de bien tristes populations urbaines : à faible coût, cette exotique proximité donne la vie. Elle introduit aussi à la chair même de l'histoire, celle de la survie alimentaire et des soins du corps, une histoire matérielle moins banale qu'en apparence car si la botanique (plus directement abordable que les vieux parchemins) a de quoi passionner petits et grands, elle n'en dépend pas moins de choix humains capitaux pour son devenir : ses multiples usages possibles - aujourd'hui oubliés du grand nombre - en font un enjeu patrimonial et l'objet d'un savoir à reconquérir. C'est à cette intersection, entre large public et spécialistes, que se situe cette publication luxueuse sur les « fruits, légumes et autres herbes de Charlemagne »: un guide descriptif et " historique " des 89 plantes dont la célèbre ordonnance carolingienne sur l'organisation des domaines royaux (avant d'être impériaux) fournit la liste dans son soixante-dixième et ultime chapitre. L'ouvrage prolonge ainsi l'ouverture il y a huit ans sur le site de l'ancienne léproserie de Melaten, près d'Aix-laChapelle - c'était bien le moins pour l'ancienne capitale impériale - d'un jardin où ces espèces ont été rassemblées dans un agréable quadrilatère suivant l'ordre même de l'énumération. 
Connu par un unique manuscrit de la bibliothèque de Wolfenbüttel (Helmstadt 254), le capitulaire De villis [vel curtis imperii] a fait couler beaucoup d'encre depuis sa redécouverte humaniste due à Matthias Flacius Illyricus au XVIe s. et sa première publication en 1647. Suscitant débat : à la fois sur sa date car il y est question de la reine à quatre reprises - or Charlemagne est veuf à partir de juin 800 -, et sur son attribution car, partant de certaines espèces citées qu'il imaginait propres à la sphère méridionale (coloquinte, pin, laurier), l'historien viennois Alphons Dopsch (Die Wirtschaftsentwicklung der Karolingerzeit, Weimar, 1912, I), avait cru en justifier la paternité à Louis, roi des Aquitains entre 794 et son association à l'empire en 813, ce qui tombait bien puisque les Annales anonymes dites de l'Astronome rapportaient la mauvaise administration de ses domaines après la famine de 792-793 et l'envoi de missi pour y remédier... mais en restreignait du même coup la portée géographique ! En 1923 dans la Revue historique (t. 143, p. 40-56), Marc Bloch réduisait ces arguments en laissant le champ le plus largement ouvert, ce qui n'empêcha pas la poursuite polémique (W. von Wartburg, A. Verhulst ou encore F.L. Ganshof). En 1985, Rüdiger Stelling (Sources. Travaux historiques, 2, p 35-41) a présenté une synthèse qui montrait qu'aucune région de l'empire ne pouvait être écartée de son application sur de stricts critères botaniques et climatologiques : d'autant que rien n'empêchait, même à l'époque carolingienne, une culture partielle sous abri ou en pots! Et s'il fallait en vérifier définitivement la validité par l'expérimentation, la réussite actuelle d'Aix-laChapelle, certes en-deçà du Rhin, fournirait une réponse des plus convaincantes.

3 La partie introductive de l'ouvrage fournit un aperçu hélas trop sommaire et mal référencé de cette historiographie (seule une bibliographie succincte figure en fin d'ouvrage, p. 415, oublieuse des références majeures et de nombreux auteurs par ailleurs aléatoirement cités dans le catalogue des espèces, Kurt Sprengel ou Ernst Meyer, pour s'en tenir aux historiens germaniques de la botanique), se contentant d'une analyse de l'ensemble des prescriptions foncières (Landgüterverordnung) et d'un aperçu sur les « gardiens de l'art de guérir » depuis l'Antiquité jusqu'à Darwin : en dépit de toute chronologie ou tradition des textes, puisque Dioscoride précède Hippocrate et Théophraste succède à Galien, sans un seul mot (ni indication de titre) sur l'Herbarius du Pseudo-Apulée ou le Tacuinum sanitatis dont les planches reproduites du somptueux manuscrit de la Bibliothèque nationale de France (fin du XIVe s.) ornent pourtant à foison le catalogue des plantes. Ce dernier constitue la part majeure et centrale de ce livre ( $\mathrm{p}$ 47-384) : le "portrait » de chaque espèce ou variété est brossé, dans l'ordre de la visite. Outre de belles photographies de leurs fleurs qui ne correspondent pas toujours, loin de là, aux utilisations ou à une description botanique suffisante - c'est un mésusage courant en la matière -, une fiche d'identification systématique chapeaute chaque entrée avec une louable traduction des dénominations courantes en anglais, français et néerlandais (mais pourquoi pas en italien ou en espagnol à l'heure européenne ? «Camomille» ne comprend pas de «h», p. 223). Des alternatives sont aussi proposées : pour le fasiolum - la dolique ou mongette -, par exemple, ou pour la solsequia (ou solsequium), qui « suit le soleil », certes, mais dont on doutera du rapprochement avec l'héliotrope au profit du souci - calendula - plus simplement conforme à l'étymon latin, ou encore pour la livèche (levisticum) où il semble quand même bien sophistiqué de vouloir aller chercher la variété alpine de la mutelline. Diverses attributions apparaitront pour le moins contestables : la renouée bistorte (polygonum) est ainsi donnée pour la dragantea, alors que Sprengel y reconnaissait l'estragon (artemisia dracunculus), ici seulement proposé en alternative, 
pour laquelle l'hypothèse de Benjamin Guérard (grand absent de ce travail, malgré son commentaire fondamental dans la Bibliothèque de l'École des chartes, XIV, 1853, p. 548-557) optait pour la serpentaire (arum dracunculus). On s'arrêtera encore sur le diptamnum vu comme fraxinelle alors que seuls les modernes l'ont dénommé dictamne blanc et qui renvoie plus sûrement à l'origan (origanum dictamnus). Pourquoi se limiter à l'unique radis (raphanus sativus) pour les radices, appellation plurielle susceptible de recouvrir d'autres raves ou plus sûrement le raifort ou radis noir (amoracia rusticana) encore fort prisé en Allemagne comme condiment ? Et que dire du silum donné exclusivement pour le sermontain (laserpitium siler), certes une apiacée fort proche mais plutôt montagnarde, alors que le choix du séseli, de la berle, ou du plaintain (psyllium) aurait été plus immédiat ? On pourra aisément disserter des types de menthe retenus (feuilles rondes ou longues, etc.), des cressons (aquatiques ou de terre) et autres courges... Et quitte à proposer des alternatives, pourquoi ne pas avoir aussi tenu compte des espèces « utilitaires » mentionnées dans les autres parties du capitulaire $(\$ 43,44,62)$ : le millet, le panic, les navets, le lin, la guède, la cardère (que l'on différenciera des autres chardons du chapitre 70), le chanvre, la mûre (qui n'est pas le fruit du mûrier), la vigne, les multiples variétés de blé, ou les arbres greffés ? Peutêtre parviendra-t-on un jour à préciser les variétés de pommiers indiquées (Gozmaringa, Geroldinga, Crevedella, Sperauca) ? La recherche eût été... fructueuse !

4 Certains de ces doutes sont du reste exposés avant la description de chaque espèce. Suivent quelques considérations archéobotaniques, et un exposé des usages et de la valeur (Nutzung und Bedeutung) accordés à la plante, du passé au présent. Cette dernière tentative, impressionniste plutôt que réellement scientifique, est la plus critiquable : il est dommage que les autorités évoquées dans la présentation générale n'aient pas été systématiquement convoquées. Pline l'Ancien en particulier, dont le livre XX de l'Histoire naturelle, spécialement consacré à la flore, si prolixe sur les multiples propriétés accordées aux simples et parfaitement connu des savants carolingiens, n'est, sous réserve d'inventaire, qu'une seule fois mentionné à propos des radis (p. 280). Caton et son traité Sur l'agriculture et Serenus Sammonicus l'auraient heureusement complété, de même qu'Apicius pour le registre culinaire. Théophraste est sollicité mais, hormis le puledium (menthe pouliot, p. 167), l'olicetum (maceron, p. 170) et l'arroche (p. 254), exclusivement pour les arbres. De même est-on étonné que les propos d'Isidore de Séville ou Hraban Maur, les « grandes " références de la culture carolingienne, soient ignorés : leur vision étymologique et symboliste aurait fourni une meilleure clé d'entrée à la polysémie spirituelle ou morale de l'Écriture sainte associée à l'univers de la Création que d'inégaux renvois directs à la Bible prise comme seul témoin d'antériorité. Le Praeceptor Germaniae est du reste loin de se limiter aux seules sphères métaphysiques (voir notre « Hraban Maur et la médecine carolingienne » in : J.L. Perrin et S. Lebecq (dir.), Hraban Maur et son temps, Colloque des Universités de Lille et Amiens, 6-8 juillet 2006, Turnhout, Brepols, 2008) et il eût été intéressant de scruter le livre XIX de sa vaste encyclopédie dont les chapitres 3, 7 et 8 ont pour objets précis les légumes, les arbres et les simples...

5 Pourquoi Avicenne (connu en Occident seulement après 1185) n'est-il cité qu'à propos de l'estragon (p. 122) ou de la laitue vireuse (p. 153) ? Pourquoi l'incontournable Hildegarde de Bingen, fort avisée de toutes les salades, disparait-elle du chapitre de la roquette (eruca), précisément là où son jugement négatif s'avère le plus savoureux ? Une nomenclature un tant soit peu rigoureuse des vertus historiquement prêtées à chacune de ces plantes par les auteurs antiques et médiévaux devra un jour 
nécessairement être dressée. Ses données pourront se voir confrontées aux compositions pharmaco-chimiques aujourd'hui reconnues en fonction d'une grille standardisée et classiquement admise (digestive, purgative, diurétique, emménagogue, carminative, tonicardiaque, hémostatique, etc.), tenant éminemment compte des parties utilisées (feuille, tige, fleur, racine, graines etc.) et de leurs modes de préparation et d'administration. Elle conduirait sans doute à mieux saisir la finalité de cette «terrible petite phrase " comme la jugeait Marc Bloch : "Nous voulons qu'au jardin on ait toutes les plantes ». Toutes ? Et pourquoi spécialement celles-ci ? S'agissait-il d'en suivre strictement l'ordre pour n'établir qu'« une sorte d'exposition horticole»?

6 Les publications du Reader's digest ou les jolis recueils publiés chez Gründ dans la défunte République démocratique restent finalement plus riches d'enseignements qu'un livre qui finit par totalement déraper en suggérant des recettes aux tomates et à la banane (p. 386, 397). Sic. Charlemagne rock'n roll ? À la banane... Pauvre Humboldt !

7 François-Olivier Touati (Université de Tours) 